\title{
Effects of Grafting on Photosynthetic Characteristics of Potential Cd-Hyperaccumulator Solanum photeinocarpum under Cd Stress
}

\author{
Fuyi Tang ${ }^{1, a}$, Lijin Lin ${ }^{2, b}$, and Ming'an Liao ${ }^{1, c^{*}}$ \\ ${ }^{1}$ College of Horticulture, Sichuan Agricultural University, Chengdu, Sichuan, China \\ ${ }^{2}$ Institute of Pomology and Olericulture, Sichuan Agricultural University, Chengdu, Sichuan, China \\ atfy1961@163.com, 'llj800924@163.com, 'Iman@sicau.edu.cn \\ ${ }^{*}$ corresponding author
}

\begin{abstract}
Keywords: Grafting; Photosynthetic characteristics; Solanum photeinocarpum; Cd stress
Abstract: A pot experiment was conducted to study the effects of grafting on photosynthetic characteristics of potential Cd-hyperaccumulator Solanum photeinocarpum under Cd stress. Four treatments were used in the experiment: ungrafted (UG), self-rooted grafting by the same $S$. photeinocarpum seedling (SG), self-rooted grafting by two different sizes of S. photeinocarpum seedlings (DG) and grafting on the rootstock of wild potato (PG). The results showed that SG and DG enhanced net photosynthetic rate (Pn) of S. photeinocarpum (Pn of DG was higher than SG), but PG decreased that. The light use efficiency (LUE) was the same as Pn. The grafting treatment decreased transpiration rate $(\mathrm{Tr})$, stomatal conductance $(\mathrm{Gs})$ and $\mathrm{CO}_{2}$ concentration of intercellular $(\mathrm{Ci})$ of $S$. photeinocarpum, and increased water use efficiency (WUE) of S. photeinocarpum. Therefore, grafting could use to enhance the photosynthetic ability of Cd-hyperaccumulator S. photeinocarpum for the phytoremediation.
\end{abstract}

\section{Introduction}

Grafting is a method which has used widely in plant propagation of horticulture, and plays a significant role in improving the yield and quality of fruit and vegetables [1]. Under heavy metal stress, the photosynthetic enzymes activity and chlorophyll synthesis are inhibited, resulting in reduced plant photosynthesis [2]. Some studies show that grafting can enhance the photosynthetic ability of plant, especially under stress condition [3-4]. So, if we use grafting method on the heavy metal hyperaccumulator plant, the photosynthetic ability of hyperaccumulator plant could enhance, and the phytoremediation ability of hyperaccumulator could improve too. Solanum photeinocarpum is a potential Cd-hyperaccumulator that grows rapidly and has a strong propagative ability [5]. To further enhancing the phytoremediation of $S$. photeinocarpum, a pot experiment was conducted to study the effects of grafting on photosynthetic characteristics of potential Cd-hyperaccumulator Solanum photeinocarpum under Cd stress. The objectives of this study were to determine whether grafting could enhance photosynthetic ability of $S$. photeinocarpum after Cd exposure.

\section{Materials and Method}

Materials. The seeds of $S$. photeinocarpum were collected from a $S$. photeinocarpum plant at the Ya'an campus farm of the Sichuan Agricultural University (29 59' N, $102^{\circ} 59^{\prime}$ E), China in July 2012, air-dried and stored at $4{ }^{\circ} \mathrm{C}$. The inceptisol soil samples (purple soil in the Genetic Soil Classification of China) were collected from the Ya'an campus farm. The basic properties of the soil were the same as reference [6], and the total Cd content was $0.101 \mathrm{mg} \cdot \mathrm{kg}^{-1}$.

Grafting. The seeds of S. photeinocarpum were sown in the farmland of the Ya' an campus farm. When the $S$. photeinocarpum seedlings reached a height of $\sim 10 \mathrm{~cm}$ (eight expanded euphyllas, rapid growth stage), the grafting was conducted. The grafting method was cleft grafting bound with $1-\mathrm{cm}$-wide plastic film. All of the leaves of the rootstocks remained. There were four grafting treatments in the experiment. (1) ungrafted (UG): S. photeinocarpum seedlings remained untreated. (2) Self-rooted grafting by the same $S$. photeinocarpum seedling (SG): The scion and rootstock were 
from the same $S$. photeinocarpum seedling. The lower parts of the $S$. photeinocarpum seedlings, with heights of $6 \mathrm{~cm}$, were the rootstocks, and the upper parts of the same $S$. photeinocarpum seedlings, with heights of $4 \mathrm{~cm}$, were the scions. The scions and rootstocks were consistent in physiology. (3) Self-rooted grafting by two different sizes of $S$. photeinocarpum seedlings (DG): The scion and rootstock were from two different growth stages of $S$. photeinocarpum seedlings. The rootstocks of the $S$. photeinocarpum seedlings were the same as in the SG, and the lower parts, with heights of $6 \mathrm{~cm}$ (10 cm for the whole plant), were the rootstocks. The scions were the upper parts $(4 \mathrm{~cm})$ of $S$. photeinocarpum seedlings with $5 \mathrm{~cm}$ of whole plant (four expanded euphyllas). The scions and rootstocks were not consistent in physiology. (4) Grafting on rootstock of wild potato (PG): Wild potato seedlings, with heights of $10 \mathrm{~cm}$, were collected from the Ya'an campus farm. The lower part of the wild potato seedlings, with heights of $6 \mathrm{~cm}$, were the rootstocks, and the upper parts of the $S$. photeinocarpum seedlings, with heights of $4 \mathrm{~cm}$, were the scions. The scions of $S$. photeinocarpum seedlings were the same as in the SG. The scions and rootstocks were not consistent in physiology. When the grafting was completed, the soil moisture content was maintained at $80 \%$ of field capacity, and all of the seedlings were covered with transparent plastic film and a shade net. After $10 \mathrm{~d}$, the transparent plastic film, the shade net and the plastic binding films were removed, and all the germinating buds of rootstocks were also removed.

Experimental Design. The experiment was conducted at the Ya' an campus farm from April to June 2013. The soil samples were air-dried and passed through a 5-mm mesh in April 2013, and then $3.0 \mathrm{~kg}$ of soil was weighed into each polyethylene pot (15 cm tall, $18 \mathrm{~cm}$ diameter). Cd was added to make a final soil $\mathrm{Cd}$ concentration of $60 \mathrm{mg} \cdot \mathrm{kg}^{-1}$ with a saturated heavy metal solution in the form of $\mathrm{CdCl}_{2} \cdot 2.5 \mathrm{H}_{2} \mathrm{O}$. The soils were mixed immediately and again after 4 weeks, during which soil moisture was kept at $80 \%$. Four uniformly prepared S. photeinocarpum seedlings of each treatment were transplanted into each pot in May 2013. Each treatment was repeated three times with a 10-cm spacing between pots. The soil moisture content was maintained at $80 \%$ of field capacity until the plants were harvested. After $S$. photeinocarpum matured (30 d of cultivation at the fully blooming stage), the photosynthesis of each plant was determined by using LI-6400 portable photosynthesis meter (LI-COR Inc., USA). The photosynthetic parameters of the photosynthesis meter were manual control $\mathrm{CO}_{2}$ concentration $400 \mu \mathrm{mol} \cdot \mathrm{CO}_{2} \mathrm{~mol}^{-1}$, temperature $25 \square$, light intensity $1200 \mu \mathrm{mol} \mathrm{m} \mathrm{m}^{-2} \cdot \mathrm{s}^{-1}$. The determination of photosynthetic parameters were net photosynthetic rate $(\mathrm{Pn})$, transpiration rate $(\mathrm{Tr})$, stomatal conductance $(\mathrm{Gs})$ and $\mathrm{CO}_{2}$ concentration of intercellular $(\mathrm{Ci})$, and each treatment was repeated three times. Water use efficiency $(\mathrm{WUE})=$ net photosynthetic rate $(\mathrm{Pn}) /$ transpiration rate (Tr), Light use efficiency $(\mathrm{LUE})=$ net photosynthetic rate $(\mathrm{Pn}) /$ light intensity [7].

\section{Results and Discussion}

Net Photosynthetic Rate (Pn). Compared with UG, SG and DG enhanced Pn of S. photeinocarpum under Cd stress, but PG decreased that (Fig. 1). The Pn of S. photeinocarpum was ranked as: DG > SG $>\mathrm{UG}>\mathrm{PG}$. SD and DG enhanced Pn of S. photeinocarpum by $15.23 \%(p<0.05)$ and $25.82 \%(p<$ $0.05)$ respectively, compared with UG, and PG decreased by 4.64\% ( $p>0.05)$ compared with UG. Because of the physiological resistance generated by the graft healing process could continue during the growth and development of the scion [8-9], the Pn of SD and DG enhanced under Cd stress, and the resistance of SG to Cd stress was lower than that of DG because of the differences in physiology when grafting. For PG, because wild potato and S. photeinocarpum were two different species, and the affinity of wild potato was lower than that of $S$. photeinocarpum as the rootstock of $S$. photeinocarpum. So, the Pn of PG was the minimum of treatments.

Transpiration Rate (Tr). Compared with UG, SG, DG and PG decreased the $\operatorname{Tr}$ of $S$. photeinocarpum (Fig. 2). The Tr of $S$. photeinocarpum was ranked as: UG $>\mathrm{SG}>\mathrm{DG}>\mathrm{PG}$. This result was in contrast to Pn, which was consistent with other studies [3-4]. SG, DG and PG decreased the $\operatorname{Tr}$ of $S$. photeinocarpum by $39.34 \%(p<0.05), 44.85 \%(p<0.05)$ and $62.13 \%(p<0.05)$ respectively, compared with UG. 


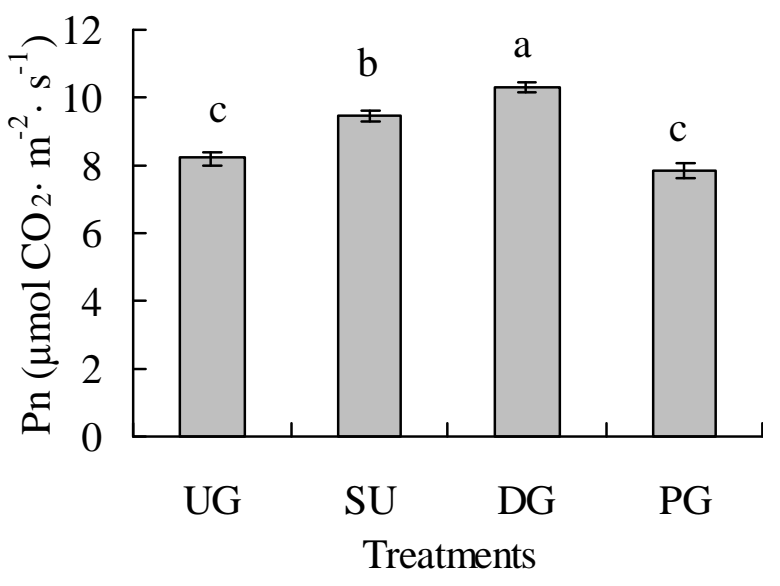

Fig. 1 Pn of grafted S. photeinocarpum

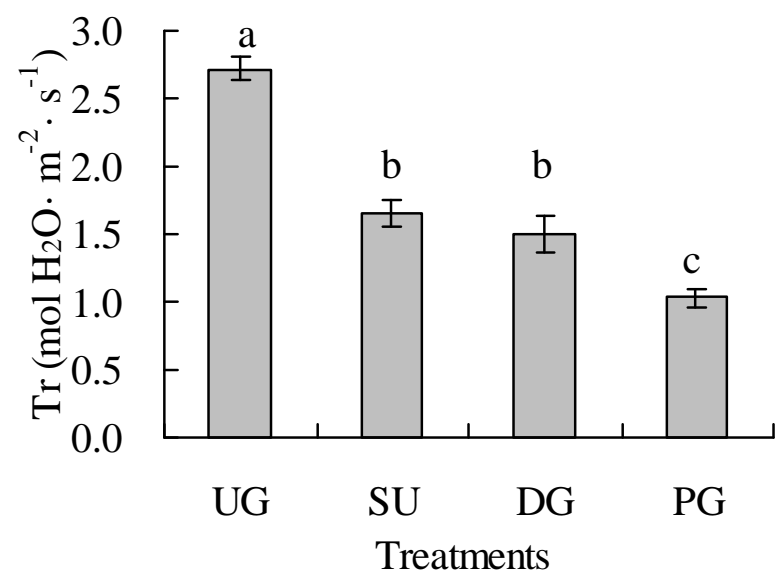

Fig. 2 Tr of grafted S. photeinocarpum

Water Use Efficiency (WUE). The Fig. 3 shows that WUE of $S$. photeinocarpum increased by grafting treatment under Cd stress. Compared with UG, SG, DG and PG increased WUE of $S$. photeinocarpum by $90.06 \%(p<0.05), 127.48 \%(p<0.05)$ and $150.66 \%(p<0.05)$ respectively, indicating that grafting could effectively improve the utilization of water, which was consistent with other studies [3-4].

Light Use Efficiency (LUE). The same as Pn, SG and DG increased LUE of S. photeinocarpum under Cd stress, but PG decreased that (Fig. 4). The LUE of S. photeinocarpum was ranked as: DG > SG > UG > PG. SG, DG and PG increased LUE of $S$. photeinocarpum by $15.17 \%(p<0.05)$ and $25.96 \%(p<0.05)$ respectively, compared with UG, and PG decreased by $4.57 \%(p>0.05)$ compared with UG.

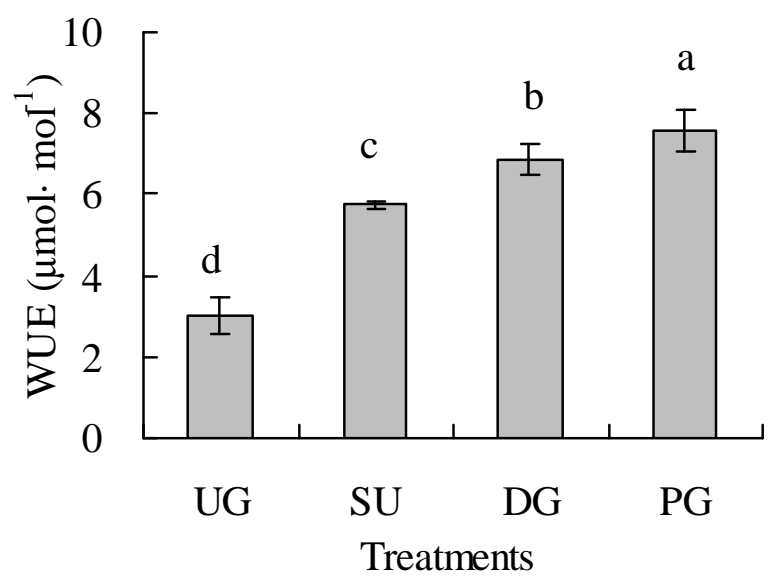

Fig. 3 WUE of grafted S. photeinocarpum

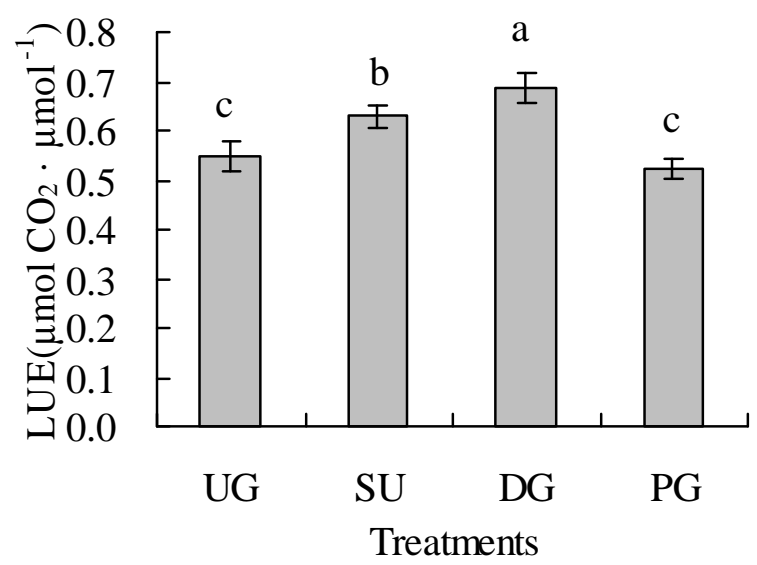

Fig. 4 LUE of grafted $S$. photeinocarpum

Stomatal Conductance (Gs). After grafting, Gs of S. photeinocarpum decreased (Fig. 5), which was benefit to Pn. The Gs of S. photeinocarpum was ranked as: UG > SG > DG > PG. Compared with UG, SG, DG and PG decreased Gs of S. photeinocarpum by $7.57 \%(p>0.05), 29.73 \%(p<0.05)$ and $50.27 \%(p<0.05)$ respectively.

$\mathrm{CO}_{2}$ Concentration of Intercellular (Ci). The same as Gs, after grafting, $\mathrm{Ci}$ of $S$. photeinocarpum decreased (Fig. 6). The Ci of S. photeinocarpum was ranked as: UG > SG > PG > DG. Compared with UG, SG, DG and PG decreased Ci of S. photeinocarpum by $0.73 \%(p>0.05), 15.87 \%(p<0.05)$ and $15.45 \%(p<0.05)$ respectively.

\section{Conclusions}

Using the same species to graft could enhance Pn of S. photeinocarpum, and Pn of DG was higher than SG under Cd stress. The wild potato as rootstock decreased Pn of S. photeinocarpum. The result of 
LUE was the same as Pn. Grafting decreased Tr, Gs and Ci of S. photeinocarpum, and increased WUE of S. photeinocarpum. Therefore, grafting could use to enhance the photosynthetic ability of Cd-Hyperaccumulator $S$. photeinocarpum, which would help to improve the phytoremediation ability of $S$. photeinocarpum.

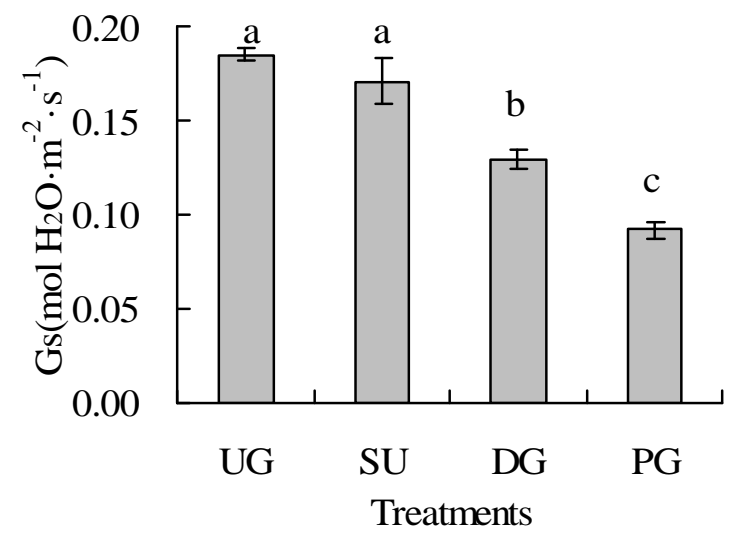

Fig. 5 Gs of grafted S. photeinocarpum

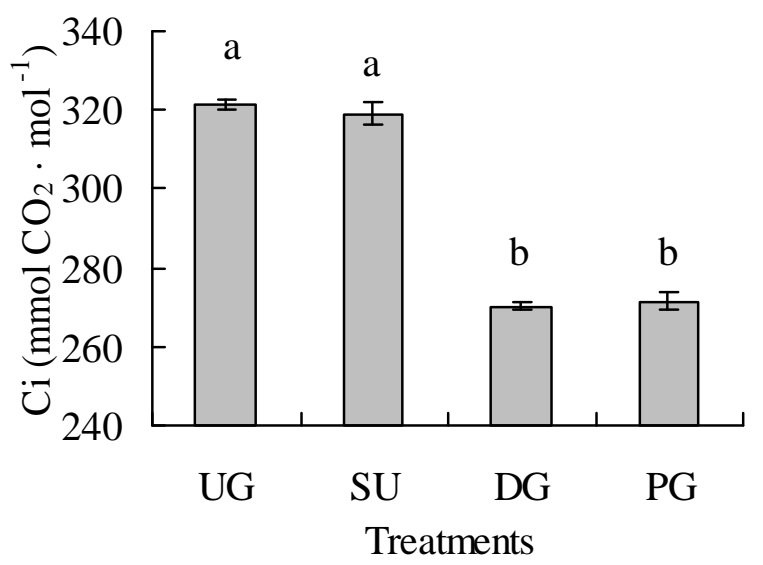

Fig. $6 \mathrm{Ci}$ of grafted S. photeinocarpum

\section{References}

[1] Y. Rouphael, D. Schwarz, A. Krumbein, G. Colla, Impact of grafting on product quality of fruit vegetables, Scientia Horticulturae 127 (2010) 172-179.

[2] M. Pal, E. Horvath, T. Janda, E. Páldi, G. Szalai, Physiological changes and defense mechanisms induced by cadmium stress in maize, Journal of Plant Nutrition and Soil Science 169 (2006) 239-246.

[3] G.L. Chen, L.C. Nie, J.W. Li, K. Xiao, Effects of low temperature stress on photosynthetic characteristics of grafted Zucchini seedlings, Acta Agriculturae Shanghai 16 (2000) 42-45 (In Chinese).

[4] H.Y. Liu, Z.J. Zhu, Q.H. Shi, Effects of low temperature stress on characteristics of photosynthesis in leaves of own-rooted and grafted watermelon seedling, Journal of Shihezi University (Natural Science) 25 (2007) 163-167 (In Chinese).

[5] X.F. Zhang, H.P. Xia, Z.A. Li, P. Zhuang, B. Gao, Identification of a new potential Cd-hyperaccumulator Solanum photeinocarpum by soil seed bank-metal concentration gradient method, Journal of Hazardous Materials 189 (2011) 414-419.

[6] L.J. Lin, Q.H. Liu, J. Shi, J.L. Sun, M.A. Liao, L.Y. Mei, Intercropping different varieties of radish can increase cadmium accumulation in radish, Environmental Toxicology and Chemistry 33 (2014) 1950-1955. 
[7] X.J. Jiang, H. Wang, W. Peng, Y.X. Yang, X.M. Zhu, L.J. Lin, Effect of compound pollution of $\mathrm{Zn}$ and $\mathrm{Cr}$ on photosynthetic physiological ccology of rice, Shanxi Journal of Agricultural Sciences 54 (2008) 56-58, 80 (In Chinese).

[8] Y. Su, J.M. Guo, Y.Q. Hu, X.W. Huo, Y.M. Chen, S.M. Cui, Effects of cicatrization on the anatomical structures and analysis of the activities of isozymes peroxidase (POD) in grafted cucumber seedlings, Journal of Shenyang Agricultural University 37 (2006) 343-347 (In Chinese).

[9] Y.Q. Hu, Y. Su, F.Y. Han, M.L. Shu, S.M. Cui, The research of anatomical structures and three activity of antioxidases change of grafted cucumber seedling, Journal of Inner Mongolia Agricultural University (Natural Science Edition) 28 (2007) 224-230 (In Chinese). 